\title{
ON SOLUTIONS TO NONHOMOGENEOUS ALGEBRAIC DIFFERENTIAL EQUATIONS AND THEIR APPLICATION
}

\author{
LIANG-WEN LIAO ${ }^{\bowtie}$ and ZHUAN YE
}

(Received 31 March 2014; accepted 10 July 2014; first published online 24 September 2014)

\author{
Communicated by P. Fenton
}

\begin{abstract}
We consider solutions to the algebraic differential equation $f^{n} f^{\prime}+Q_{d}(z, f)=u(z) e^{v(z)}$, where $Q_{d}(z, f)$ is a differential polynomial in $f$ of degree $d$ with rational function coefficients, $u$ is a nonzero rational function and $v$ is a nonconstant polynomial. In this paper, we prove that if $n \geq d+1$ and if it admits a meromorphic solution $f$ with finitely many poles, then

$$
f(z)=s(z) e^{\nu(z) /(n+1)} \quad \text { and } \quad Q_{d}(z, f) \equiv 0 .
$$

With this in hand, we also prove that if $f$ is a transcendental entire function, then $f^{\prime} p_{k}(f)+q_{m}(f)$ assumes every complex number $\alpha$, with one possible exception, infinitely many times, where $p_{k}(f), q_{m}(f)$ are polynomials in $f$ with degrees $k$ and $m$ with $k \geq m+1$. This result generalizes a theorem originating from Hayman ['Picard values of meromorphic functions and their derivatives', Ann. of Math. (2) 70(2) (1959), 9-42].
\end{abstract}

2010 Mathematics subject classification: primary 34M05; secondary 34M15, 30D35.

Keywords and phrases: nonlinear differential equations, entire solutions, meromorphic solutions, Nevanlinna theory, normal family, Picard values.

\section{Introduction and main results}

Let $\Lambda=\left\{\left(\lambda_{0}, \lambda_{1}, \ldots, \lambda_{\mu}\right): \lambda_{j}\right.$ is a nonnegative integer and $\left.0 \leq j \leq \mu<\infty\right\}$ be an index set with a finite cardinal number and let

$$
Q_{d}(z, f)=\sum_{\lambda \in \Lambda} a_{\lambda}(z) f^{\lambda_{0}}\left(f^{\prime}\right)^{\lambda_{1}} \cdots\left(f^{(\mu)}\right)^{\lambda_{\mu}}
$$

be a polynomial of $f$ and its derivatives with degree $d$ and meromorphic function coefficients $a_{\lambda}(z)$, where $d:=\operatorname{deg}(Q(z, f))=\max _{\lambda \in \Lambda} \sum_{j=0}^{\mu} \lambda_{j}$. In the sequel, we simply call $Q_{d}(z, f)$ a differential polynomial of $f$ with degree $d$. We also assume that the reader is familiar with the simple standard notation and results in Nevanlinna theory

The research was supported by NSF of China (Grant 11271179).

(C) 2014 Australian Mathematical Publishing Association Inc. 1446-7887/2014 \$16.00 
(see [3, 8]). The term $S(r, f)$ always has the property that $S(r, f)=o(T(r, f))$ as $r \rightarrow \infty$, possibly outside a set $E$ of finite linear measure. The set $E$ is not necessarily the same at each occurrence. A meromorphic function $a(z)$ is said to be a small function with respect to $f(z)$ if and only if $T(r, a)=S(r, f)$.

It is always an essential problem to find the structure of solutions to any differential equation. In 1980, Gackstatter and Laine [6] conjectured that the algebraic differential equation

$$
\left(f^{\prime}\right)^{n}=p_{m}(f)
$$

where $p_{m}(f)$ is a polynomial in $f$ and $n$ is a positive integer, does not possess any admissible solution when $m \leq n-1$. In 1990, He and Laine [9] gave a positive answer to the conjecture. Recently, Zhang and the first author [21] proved that if the algebraic differential equation with polynomial coefficients

$$
Q_{d}(z, f)=0
$$

has only one dominant term (highest-degree term), then (1.1) has no admissible transcendental meromorphic solutions with a few poles. There are also many other papers concerning the structure of solutions to various differential equations (for example [1, 4, 10-14]). In this paper, we consider the algebraic differential equation

$$
f^{n} f^{\prime}+Q_{d}(z, f)=u(z) e^{v(z)}
$$

where $Q_{d}(z, f)$ be a differential polynomial in $f$ with $n \geq d+1$ and rational function coefficients, $u$ is a nonzero rational function and $v$ is a nonconstant polynomial. Clearly, $f^{n} f^{\prime}$ is the only dominant term in (1.2) and its nonhomogeneous term is a transcendental meromorphic function. Thus, we find a simple and neat expression for meromorphic solutions to (1.2) if the solutions have a few poles. This also means that the solution has finitely many zeros determined by the term $u e^{v}$ in the differential equation. Further, the result can be used to generalize a theorem of Hayman [7].

Hayman [7] proved that if $f$ is a transcendental entire function, then $f^{\prime} f^{n}$ assumes every nonzero complex number infinitely many times, provided that $n \geq 2$. Since then, there are many research publications $[2,5,15,16,20]$ regarding this type of Picard-value problem. For example, Mues [15] extended the result, proving that if $p(f)$ is a nonconstant polynomial in $f$, then $p(f) f^{\prime}$ assumes every nonzero complex number infinitely many times. Zhang and Li [20] proved that if $f$ is a transcendental meromorphic function with $N(r, f)=S(r, f)$ and $p$ a polynomial with degree $\geq 1$, then $p(f) f^{\prime}$ takes every nonzero complex number infinitely many times. In this paper, we also prove that if $p_{k}, q_{m}$ are two polynomials with degrees $k \geq m+1$ and $f$ a transcendental entire function, then $p_{k}(f) f^{\prime}+q_{m}(f)$ assumes every complex number, with possibly one exceptional value, infinitely many times. More interestingly, we show that if $p_{k}(f) f^{\prime}+q_{m}(f)$ takes the exceptional value finitely many times, then we can prove that $q_{m}$ has to be a constant polynomial and $p_{n}$ is a complete power function, or $f(z)=A e^{B z}+C$, where $A, B, C$ are constant. Now we are ready to state our theorems. 
THeOREM 1.1. Let $Q_{d}(z, f)$ be a differential polynomial in $f$ of degree $d$ with rational function coefficients. Suppose that $u$ is a nonzero rational function and $v$ is a nonconstant polynomial. If $n \geq d+1$ and the differential equation

$$
f^{n} f^{\prime}+Q_{d}(z, f)=u(z) e^{v(z)}
$$

admits a meromorphic solution $f$ with finitely many poles, then $f$ has the following form:

$$
f(z)=s(z) e^{v(z) /(n+1)} \quad \text { and } \quad Q_{d}(z, f) \equiv 0,
$$

where $s(z)$ is a rational function with $s^{n}\left((n+1) s^{\prime}+v^{\prime} s\right)=(n+1) u$. In particular, if $u$ is a polynomial, then $s$ is a polynomial, too.

REMARK 1.2. The condition $n \geq d+1$ in the theorem is necessary. For example, $f(z)=e^{z}+z$ solves the following differential equation:

$$
f^{\prime} f^{2}-2 z f^{\prime 2}-z^{2} f^{\prime}=e^{3 z}
$$

where $n=d=2$.

THEOREM 1.3. Let $f$ be a transcendental entire function, $q_{m}(f)=b_{m} f^{m}+\cdots+b_{1} f+b_{0}$ a polynomial with degree $m$ and $n$ a positive integer with $n \geq m+1$. Then $f^{\prime} f^{n}+q_{m}(f)$ assumes every complex number $\alpha$ infinitely many times, except for a possible value $b_{0}=q_{m}(0)$. On the other hand, if $f^{\prime} f^{n}+q_{m}(f)$ assumes $b_{0}=q_{m}(0)$ finitely many times, then $q_{m}(z) \equiv b_{0}, f$ and $f^{\prime}$ have only finitely many zeros.

REMARK 1.4. The restrictive condition $n \geq m+1$ in the theorem is necessary. For instance, if $f(z)=e^{z}+1$ and $q_{2}(z)=-2 z^{2}+3 z$, then $f^{\prime} f^{2}-2 f^{2}+3 f=e^{3 z}+1$ does not assume $1 \neq q_{2}(0)$.

THeorem 1.5. Let $f$ be a transcendental entire function, $p_{n}(f)=a_{n} f^{n}+\cdots+a_{0} a$ polynomial with degree $n, q_{m}(f)=b_{m} f^{m}+\cdots+b_{0}$ a polynomial with degree $m$ and $n \geq m+1$. Then $f^{\prime} p_{n}(f)+q_{m}(f)$ assumes every complex number $\alpha$ infinitely many times, except for a possible value $q_{m}\left(-a_{n-1} / n a_{n}\right)$. On the other hand, if $f^{\prime} p_{n}(f)+$ $q_{m}(f)$ assumes the complex value $q_{m}\left(-a_{n-1} / n a_{n}\right)$ finitely many times, then either:

(1) $p_{n}(z)=a_{n}\left(z+a_{n-1} / n a_{n}\right)^{n}, q_{m}(z)$ is a constant polynomial, which is $q_{m}\left(-a_{n-1} / n a_{n}\right)$; and $f+a_{n-1} / n a_{n}, f^{\prime}$ have only finitely many zeros; or

(2) $f(z)=A e^{B z}+a_{n-1} / n a_{n}$, where $A, B$ are some constants, only when $q_{m}$ is nonconstant and $f$ is of finite order.

REMARK 1.6. Theorem 1.3 is a special case of Theorem 1.5. But, we need Theorem 1.3 in the proof of Theorem 1.5. Otherwise, we think the proof will be too long if we combine Theorems 1.3 and 1.5 together.

REMARK 1.7. It is challenge to prove that Theorem 1.3 and/or Theorem 1.5 are valid for meromorphic functions in the complex plane.

EXAmple 1.8. If $f(z)=e^{z}$, then $f^{\prime}\left(f^{3}-f\right)+f^{2}=e^{3 z}$ does not assume $0=q_{2}(0)$. If $g(z)=e^{e^{z}}+1$, then $g^{\prime}(g-1)^{n}$ does not assume zero. 


\section{Lemmas}

Lemma 2.1 (Clunie lemma [5, 17]). Let $f(z)$ be a transcendental meromorphic function in the complex plane and satisfying

$$
f^{n}(z) P(z, f)=Q(z, f),
$$

where $P(z, f), Q(z, f)$ are differential polynomials with rational function coefficients. If the degree of $Q(z, f)$ is at most $n$, then

$$
m(r, P(z, f))=O(\log r)(r \rightarrow \infty),
$$

if $f$ is of finite order, and

$$
m(r, P(z, f))=O(\log (r T(r, f)))(r \rightarrow \infty),
$$

possibly outside a set $E$ of $r$ with finite linear measure, if $f(z)$ is of infinite order.

Lemma 2.2 (Borel lemma [18]). Let $a_{j}(z)$ be entire functions of finite order $\leq \rho$. Let $g_{j}(z)$ be entire and $g_{k}(z)-g_{j}(z)(j \neq k)$ be a transcendental entire function or polynomial of degree greater than $\rho$. Then

$$
\sum_{j=1}^{n} a_{j}(z) e^{g_{j}(z)}=a_{0}(z)
$$

holds only when

$$
a_{0}(z)=a_{1}(z)=\cdots=a_{n}(z) \equiv 0 .
$$

Lemma 2.3. Let $n \geq 2$ be an integer and $P_{d}(z, f)$ denote an algebraic differential polynomial in $f(z)$ of degree $d \leq n-1$ with small functions of $f$ as its coefficients. If $p(z)$ is a small function of $f$, if $\alpha$ is a nonconstant polynomial and if $f$ is a meromorphic solution of the equation

$$
f^{n}(z) f^{\prime}(z)+P_{d}(z, f)=p(z) e^{\alpha(z)}
$$

and $N(r, f)=S(r, f)$, then $f$ is of finite order.

Proof. Clearly, any meromorphic function satisfying the equation in the lemma must be transcendental. Denote $k=\operatorname{deg} \alpha$. By the Clunie lemma and $N(r, f)=S(r, f)$,

$$
\begin{aligned}
(n+1) T(r, f) & =T\left(r, f^{n+1}\right)=T\left(r, \frac{1}{f^{n+1}}\right)+S(r, f) \\
& =m\left(r, \frac{1}{f^{\prime} f^{n}}\right)+m\left(r, \frac{f^{\prime}}{f}\right)+N\left(r, \frac{1}{f^{\prime} f^{n}}\right)+N\left(r, \frac{1}{f}\right)-N\left(r, \frac{1}{f^{\prime}}\right)+S(r, f) \\
& \leq T\left(r, f^{\prime} f^{n}\right)+N\left(r, \frac{1}{f}\right)+S(r, f)=m\left(r, f^{\prime} f^{n}\right)+N\left(r, \frac{1}{f}\right)+S(r, f) \\
& \leq m\left(r, p(z) e^{\alpha(z)}\right)+m\left(r, P_{d}(z, f)\right)+N\left(r, \frac{1}{f}\right)+S(r, f) \\
& \leq A r^{k}+(d+1) T(r, f)+S(r, f) .
\end{aligned}
$$

Of course, the assumptions that $N(r, f)=S(r, f)$ and $N\left(r, p e^{\alpha}\right)=S(r, f)$ have been used here. Thus, $(n-d) T(r, f) \leq A r^{k}+S(r, f)$ and $f$ is of finite order. 
LEMMA 2.4. If $f$ is a meromorphic function of infinite order, then there exists a sequence $\left\{z_{k}\right\}$ with $\lim _{k \rightarrow \infty} z_{k}=\infty$ such that $\left\{f\left(z_{k}+z\right)\right\}_{k=1}^{\infty}$ is not normal at $z=0$.

Proof. First, we claim that $\limsup _{z \rightarrow \infty} f^{\#}(z)=\infty$, where $f^{\#}(z)=\left|f^{\prime}(z)\right| /\left(1+|f(z)|^{2}\right)$ is the spherical derivative of $f$. Indeed, if it is not true, then there is a positive constant $C$ such that $f^{\#}(z) \leq C$ for $z \in \mathbb{C}$. Therefore,

$$
T(r, f)=\frac{1}{\pi} \int_{0}^{r} \frac{1}{t}\left(\iint_{|z| \leq t}\left(f^{\#}(z)\right)^{2} d x d y\right) d t \leq \frac{C^{2}}{2} r^{2} .
$$

It follows that the order of $f$ is not greater than two, which contradicts the assumption that $f$ is of infinite order. Hence, there exists a sequence $\left\{z_{k}\right\}$ with $\lim _{k \rightarrow \infty} z_{k}=\infty$ such that $\lim _{k \rightarrow \infty} f^{\#}\left(z_{k}\right)=+\infty$. Set $\left\{g_{k}(z)=f\left(z_{k}+z\right)\right\}_{k=1}^{\infty}$. Then $g_{k}^{\#}(0)$ is unbounded. Therefore, by Marty's criterion, $\left\{g_{k}(z)=f\left(z_{k}+z\right)\right\}_{k=1}^{\infty}$ is not normal at $z=0$.

Lemma 2.5 (Zalcman-Pang lemma [19]). Let $\mathcal{F}$ be a family of meromorphic functions on $D=\{|z|<1\}$ and $\alpha$ a real number satisfying $-1<\alpha<1$. Then $\mathcal{F}$ is not normal in $D$ if and only if there exist:

(i) a number $r, 0<r<1$;

(ii) a sequence of points $z_{k},\left|z_{k}\right|<r$;

(iii) a positive sequence $\rho_{k}, \rho_{k} \rightarrow 0$; and

(iv) a sequence $\{f\}_{n \in N} \subset \mathcal{F}$ such that $\rho_{k}^{\alpha} f_{k}\left(z_{k}+\rho_{k} \zeta\right) \rightarrow g(\zeta)$ spherically uniformly on compact subsets of $\mathbb{C}$, where $g$ is a nonconstant meromorphic function of order $\rho(g) \leq 2$.

\section{Proofs of the theorems}

3.1. Proof of Theorem 1.1. Let $f$ be a meromorphic solution of (1.3) with finitely many poles. It follows from Lemma 2.3 that the order of $f$ is finite. If $f$ has only finitely many zeros, then $f(z)=s(z) e^{t(z)}$, where $s(z)$ is a rational function and $t(z)$ is a polynomial. By substituting $f$ into (1.3) and using Lemma 2.2, we obtain $Q_{d}(z, f) \equiv 0$, $(n+1) t(z)=v(z)$ and $s^{n}\left(s^{\prime}+t^{\prime} s\right)=u$, which also implies that $s$ is a polynomial if $u$ is a polynomial. Thus, Theorem 1.1 is proved when $f$ has only finitely many zeros. Now we assume that $f$ has infinitely many zeros. Denote $Q=Q_{d}(z, f)$. Then

$$
f^{n} f^{\prime \prime}+n f^{n-1}\left(f^{\prime}\right)^{2}+Q^{\prime}=\left(u^{\prime}+v^{\prime} u\right) e^{v(z)} .
$$

It follows from (1.3) and (3.1) that

$$
\left(u f f^{\prime \prime}+n u\left(f^{\prime}\right)^{2}-\left(u^{\prime}+v^{\prime} u\right) f f^{\prime}\right) f^{n-1}=Q_{d}^{*}(z, f),
$$

where $Q_{d}^{*}(z, f)=u Q^{\prime}-\left(u^{\prime}+v^{\prime} u\right) Q$ is a differential polynomial of $f$ with degree $d$ and rational function coefficients. It follows from Lemma 2.1 that there is a rational function $\psi$ such that

$$
u(z) f(z) f^{\prime \prime}(z)+n u(z)\left(f^{\prime}(z)\right)^{2}-\left(u^{\prime}(z)+v^{\prime}(z) u(z)\right) f(z) f^{\prime}(z)=\psi(z) .
$$


If $\psi(z) \equiv 0$, then

$$
u(z) f(z) f^{\prime \prime}(z)+n u(z)\left(f^{\prime}(z)\right)^{2}=\left(u^{\prime}(z)-v^{\prime}(z) u(z)\right) f(z) f^{\prime}(z) .
$$

Let $z_{0}$ be a zero of $f$ which is not a zero or pole of $u(z)$ and $v(z)$; then it follows from (3.3) that $f^{\prime}\left(z_{0}\right)=0$. Thus, $z_{0}$ is a zero of $f$ with multiplicity $n \geq 2$. By comparing the multiplicities at $z_{0}$ of both sides of (3.3), we have $u\left(z_{0}\right)=0$, which is a contradiction. Hence, $\psi_{(}(z) \not \equiv 0$.

By differentiating (3.2),

$u f^{\prime \prime \prime} f+(2 n+1) u f^{\prime} f^{\prime \prime}-\left(u^{\prime \prime}+v^{\prime \prime} u+v^{\prime} u^{\prime}\right) f^{\prime} f-v^{\prime} u f^{\prime \prime} f+\left((n-1) u^{\prime}+v^{\prime} u\right)\left(f^{\prime}\right)^{2}=\psi^{\prime}$.

Multiplying (3.2) by $\psi^{\prime}$ and (3.4) by $\psi$, and then subtracting the resulting equations,

$$
\left(Q_{1} f^{\prime}+Q_{2} f^{\prime \prime}+Q_{3} f^{\prime \prime \prime}\right) f=f^{\prime}\left(B_{1} f^{\prime}+B_{2} f^{\prime \prime}\right),
$$

where

$$
\begin{aligned}
& Q_{1}=\psi(z)\left(u^{\prime \prime}(z)+v^{\prime \prime}(z) u(z)+v^{\prime}(z) u(z)\right)-\psi^{\prime}(z)\left(u^{\prime}(z)+v^{\prime}(z) u(z)\right), \\
& Q_{2}=\psi^{\prime}(z) u(z)+\psi(z) v(z) u(z), \\
& Q_{3}=-\psi(z) u(z), \\
& B_{1}=(n-1) \psi(z) u^{\prime}(z)-\psi(z) v^{\prime}(z) u(z)-n \psi^{\prime}(z) u(z), \\
& B_{2}=(2 n+1) \psi(z) u(z) .
\end{aligned}
$$

If $z_{0}$ is a zero of $f(z)$, but is not a zero or pole of $u(z), v(z)$ and $\psi(z)$, then $f^{\prime}\left(z_{0}\right)^{2}=$ $\psi\left(z_{0}\right) / n u\left(z_{0}\right) \neq 0$ by (3.2). Hence, $z_{0}$ is a simple zero of $f$ and, consequently, (3.5) gives

$$
B_{1}\left(z_{0}\right) f^{\prime}\left(z_{0}\right)+B_{2}\left(z_{0}\right) f^{\prime \prime}\left(z_{0}\right)=0 \text {. }
$$

Thus, $\left(B_{1} f^{\prime}+B_{2} f^{\prime \prime}\right) / f$ has only finitely many poles and, by the logarithmic derivative lemma,

$$
T\left(r, \frac{B_{1} f^{\prime}+B_{2} f^{\prime \prime}}{f}\right)=m\left(r, \frac{B_{1} f^{\prime}+B_{2} f^{\prime \prime}}{f}\right)+O(\log r)=O(\log r) .
$$

Therefore, $A(z):=\left(B_{1} f^{\prime}+B_{2} f^{\prime \prime}\right) / f$ is a rational function and $f$ satisfies the following differential equation:

$$
B_{2} f^{\prime \prime}+B_{1} f^{\prime}-A f=0
$$

If $A \equiv 0$, then

$$
\frac{f^{\prime \prime}}{f^{\prime}}=-\frac{B_{1}}{B_{2}}=-\left(\frac{n-1}{2 n+1} \frac{u^{\prime}}{u}-\frac{n}{2 n+1} \frac{\psi^{\prime}}{\psi}-\frac{1}{2 n+1} v^{\prime}\right) .
$$

By integrating the above equation,

$$
f^{\prime}(z)=\beta_{1}(z) e^{(1 /(2 n+1)) v(z)},
$$

where $\beta_{1}(z)$ is a rational function. Substituting $f^{\prime}$ into (3.2),

$$
\beta_{2}(z) e^{(1 /((2 n+1))) v(z)} f(z)=n u(z) \beta_{1}(z)^{2} e^{(2 /((2 n+1))) v(z)}-\psi(z),
$$


where $\beta_{2}(z)$ is a nonzero rational function. Thus,

$$
f=\beta_{3}(z) e^{(1 /((2 n+1))) v(z)}+\beta_{4}(z) e^{-(1 /((2 n+1))) v(z)},
$$

where $\beta_{3}(z)=n u(z) \beta_{1}^{2} / \beta_{2}$ and $\beta_{4}(z)=-\psi / \beta_{2}$ are rational functions. It follows from (3.7) and (3.8) that

$$
\begin{array}{r}
\left(\beta_{1}(z)-\beta_{3}^{\prime}(z)-\frac{1}{2 n+1} \beta_{3}(z) v^{\prime}(z)\right) e^{(1 /((2 n+1))) v(z)} \\
=\left(\beta_{4}^{\prime}(z)-\frac{1}{2 n+1} \beta_{4}(z) v^{\prime}(z)\right) e^{-(1 /((2 n+1))) v(z)} .
\end{array}
$$

Hence, Lemma 2.2 implies that

$$
\beta_{4}^{\prime}(z)-\frac{1}{2 n+1} \beta_{4}(z) v^{\prime}(z) \equiv 0 .
$$

If $\beta_{4}(z) \not \equiv 0$, then $\beta_{4}(z)=C e^{(1 /((2 n+1))) v(z)}$, which contradicts the fact that $\beta_{4}(z)$ is a rational function. If $\beta_{4}(z) \equiv 0$, then $\psi(z) \equiv 0$, a contradiction again. Hence, $A \not \equiv 0$.

By a similar argument as we have used above, $A_{1}(z):=\left(Q_{1} f^{\prime}+Q_{2} f^{\prime \prime}+Q_{3} f^{\prime \prime \prime}\right) / f^{\prime}$ is a rational function. Thus, $f$ satisfies the following differential equation:

$$
Q_{3} f^{\prime \prime \prime}+Q_{2} f^{\prime \prime}+\left(Q_{1}-A_{1}\right) f^{\prime}=0 .
$$

By differentiating (3.6), we obtain

$$
B_{2} f^{\prime \prime \prime}+\left(B_{2}^{\prime}+B_{1}\right) f^{\prime \prime}+\left(B_{1}^{\prime}-A\right) f^{\prime}-A^{\prime} f=0 .
$$

By eliminating $f^{\prime \prime \prime}$ from (3.9) and (3.10),

$$
\left[(2 n+1) Q_{2}+B_{2}^{\prime}+B_{1}\right] f^{\prime \prime}+\left[(2 n+1)\left(Q_{1}-A_{1}\right)+B_{1}^{\prime}-A\right] f^{\prime}-A^{\prime} f=0 .
$$

It follows from (3.6) and (3.11) that

$$
R_{1} f^{\prime}+R_{2} f=0
$$

where

$$
\begin{aligned}
& R_{1}=B_{1}\left[(2 n+1) Q_{2}+B_{2}^{\prime}+B_{1}\right]-B_{2}\left[(2 n+1)\left(Q_{1}-A_{1}\right)+B_{1}^{\prime}-A\right], \\
& R_{2}=A^{\prime} B_{2}-A\left[(2 n+1) Q_{2}+B_{2}^{\prime}+B_{1}\right] .
\end{aligned}
$$

If $R_{1} \equiv 0$, then $R_{2} \equiv 0$. Hence,

$$
\frac{A^{\prime}}{A}=\frac{(2 n+1) Q_{2}+B_{2}^{\prime}+B_{1}}{B_{2}}=\frac{\psi^{\prime}}{\psi}+\frac{B_{2}^{\prime}}{B_{2}}+\frac{n-1}{2 n+1} \frac{u^{\prime}}{u}+\frac{v}{2 n+1}+\frac{v^{\prime}}{2 n+1} .
$$

Thus,

$$
\frac{v}{2 n+1}+\frac{v^{\prime}}{2 n+1}=\frac{A^{\prime}}{A}-\frac{\psi^{\prime}}{\psi}-\frac{B_{2}^{\prime}}{B_{2}}-\frac{n-1}{2 n+1} \frac{u^{\prime}}{u} .
$$

This is impossible, since the left-hand side tends to infinity and the right-side tends to zero as $z$ tends to infinity. This shows that $R_{1} \not \equiv 0$ and

$$
\frac{f^{\prime}}{f}=-\frac{R_{2}}{R_{1}} \text {. }
$$


Since $f^{\prime} / f$ has only simple poles, so does $R_{2} / R_{1}$; therefore, $f(z)=s(z) e^{v_{*}(z)}$, where $s(z)$ is a rational function and $v_{*}(z)$ is a polynomial. By substituting $f$ into (1.3) and using Lemma 2.2, we obtain $Q_{d}(z, f) \equiv 0,(n+1) v_{*}(z)=v(z)$ and $s^{n}\left(s^{\prime}+v_{*}^{\prime} s\right)=u$, which also implies that $s$ is a polynomial if $u$ is a polynomial.

3.2. Proof of Theorem 1.3. If $f$ has finite order and $f^{\prime} f^{n}+q_{m}(f)$ assumes a complex number $a \neq b_{0}$ finitely many times, then $f$ satisfies the following differential equation:

$$
f^{\prime} f^{n}+q_{m}(f)-a=u(z) e^{v(z)},
$$

where $u(z)$ and $v(z)$ are polynomials. Thus, it follows from Theorem 1.1 that there is a polynomial $s$ such that $f(z)=s(z) e^{v(z) /(n+1)}$ and $q_{m}(f)-a \equiv 0$, from which Lemma 2.2 implies $b_{0}-a=0$. This is a contradiction.

If $f$ has infinite order and $f^{\prime} f^{n}+q_{m}(f)$ assumes a complex number $\alpha$ finitely many times, then we get from Lemma 2.4 that there exists a sequence $\left\{z_{k}\right\}$ with $\lim _{k \rightarrow \infty} z_{k}=\infty$ such that $\left\{g_{k}(z)=f\left(z_{k}+z\right)\right\}_{k=1}^{\infty}$ is not normal at $z=0$. By Lemma 2.5, there exist a sequence of $\left\{w_{k}\right\}_{k=1}^{\infty}$ with $\left|w_{k}\right|<1$ and a positive sequence of $\rho_{k}$ with $\rho_{k} \rightarrow 0$ such that

$$
h_{k}(z)=\rho_{k}^{-1 /(n+1)} g_{k}\left(w_{k}+\rho_{k} z\right)=\rho_{k}^{-1 /(n+1)} f\left(w_{k}+z_{k}+\rho_{k} z\right) \rightarrow g(z)
$$

spherically uniformly on any compact subset of $\mathbb{C}$, where $g(z)$ is a nonconstant entire function and its order satisfies $\rho(g) \leq 2$. Thus,

$$
\begin{aligned}
F_{k}(z) & :=f^{\prime}\left(w_{k}+z_{k}+\rho_{k} z\right) f\left(w_{k}+z_{k}+\rho_{k} z\right)^{n}+q_{m}\left(f\left(w_{k}+k z_{k}+\rho_{k} z\right)\right) \\
& =h_{k}^{\prime}(z) h_{k}(z)^{n}+\left(b_{m} \rho_{k}^{m /(n+1)} h_{k}^{m}+\cdots+b_{1} \rho_{k}^{1 /(n+1)} h_{k}+b_{0}\right)
\end{aligned}
$$

converges to $g^{\prime}(z) g(z)^{n}+b_{0}$ spherically uniformly on any compact subset of $\mathbb{C}$. We now consider two cases.

Case 1. When $g^{\prime}(z) g(z)^{n}+b_{0}-a$ has a zero, say $\zeta_{0}$. Then, by Hurwitz's theorem, there are a sequence of complex numbers $\left\{\zeta_{k}\right\}$ and a sufficiently large integer $N$ such that $F_{k}\left(\zeta_{k}\right)-a=0(k \geq N)$ and $\zeta_{k} \rightarrow \zeta_{0}$. Hence,

$$
f^{\prime}\left(\xi_{k}\right) f\left(\xi_{k}\right)^{n}+q_{m}\left(f\left(\xi_{k}\right)\right)-a=0,
$$

where $\xi_{k}=w_{k}+z_{k}+\rho_{k} \zeta_{k}$. Since $\left|w_{k}\right|<1, z_{k} \rightarrow \infty, \rho_{k} \rightarrow 0$ and $\zeta_{k} \rightarrow \zeta_{0}$ as $k \rightarrow \infty$, we can choose subsequences $\left\{z_{k_{j}}\right\},\left\{w_{k_{j}}\right\}$ and $\left\{\rho_{k_{j}}\right\}$ such that

$$
\left|z_{k_{j}}-z_{k_{l}}\right|>3 \text { when } k_{j} \neq k_{l}, \quad\left|w_{k_{j}}\right|<1 \quad \text { and } \quad\left|\rho_{k_{j}} \zeta_{k_{j}}\right|<\frac{1}{2} .
$$

Thus, $\xi_{k_{j}}=w_{k_{j}}+z_{k_{j}}+\rho_{k_{j}} \zeta_{k_{j}}(j=1,2, \ldots)$ are distinct zeros of $f^{\prime} f^{n}-q_{m}(f)-a$, which is a contradiction.

Case 2. When $g^{\prime}(z) g(z)^{n}+b_{0}-a$ does not have a zero. Thus, $g(z)$ must be a transcendental entire function of finite order and is a solution of the differential equation

$$
g^{\prime} g^{n}+b_{0}-a=e^{v(z)}
$$


where $v(z)$ is a polynomial. It follows from Theorem 1.1 that $a-b_{0}=0$. This means that $f^{\prime} f^{n}+q_{m}(f)$ takes any complex number $\alpha \neq b_{0}=q_{m}(0)$ infinitely many times. Thus, the first part of the theorem is proved.

On the other hand, if $f^{\prime} f^{n}+q_{m}(f)$ assumes $b_{0}=q_{m}(0)$ finitely many times, then

$$
f^{\prime} f^{n}+q_{m}(f)-q_{m}(0)=f\left(f^{\prime} f^{n-1}+b_{m} f^{m-1}+\cdots+b_{1}\right)
$$

has only finitely many zeros. It follows that $f$ and $f^{\prime} f^{n-1}+b_{m} f^{m-1}+\cdots+b_{1}$ have only finitely many zeros. That is, $f^{\prime} f^{n-1}+b_{m} f^{m-1}+\cdots+b_{1}$ assumes the value 0 finitely many times. By similar arguments as we have done with $f^{\prime} f^{n}+b_{m} f^{m}+\cdots+$ $b_{1} f+b_{0}$ and $\alpha=0$ above, we have $b_{1}=\alpha=0$. Continuing the arguments, we have $b_{1}=b_{2}=\cdots=b_{m}=0$. Hence, $q_{m}(z) \equiv b_{0}$ and $f^{\prime} f^{n}$ has only finitely many zeros; so do $f$ and $f^{\prime}$. We have completed the proof.

By the proof of Theorem 1.3, we obtain the following result.

Corollary 3.1. Let $\mathfrak{F}$ be a family of holomorphic functions in a domain D. Suppose that $f^{\prime} f^{n}+q_{m}(f)$ does not assume a complex number $\alpha \neq q_{m}(0)$ for every function $f \in \mathfrak{F}$, where $n \geq 1, q_{m}(z)$ is a polynomial with degree $m$ and $m \leq n-1$. Then $\mathfrak{F}$ is a normal family in the domain $D$.

In fact, if $\mathfrak{F}$ is not normal at $z_{0}$, then it follows from Lemma 2.5 that there is a nonconstant entire function $g$ of order $\rho(g) \leq 2$ such that $g^{\prime} g^{n}$ does not assume $q_{m}(0)-\alpha \neq 0$. This is impossible.

3.3. Proof of Theorem 1.5. We first discuss the case that $f$ is a transcendental entire function of finite order. If $n=1$, then let $p_{1}(f)=a_{1} f+a_{0}$ with $a_{1} \neq 0$. Since $n \geq m+1=0, q_{m}(f)$ is a constant, say $b_{0}$. If $f^{\prime} p_{1}(f)+b_{0}$ assumes $\alpha$ finitely many times, then there are a polynomial $s$ and a nonconstant polynomial $v$ such that

$$
f^{\prime}(z) p_{1}(f(z))+b_{0}-\alpha=s(z) e^{v(z)} .
$$

Set $g(z)=f(z)+a_{0} / a_{1}$. Then

$$
g^{\prime} g=\frac{f^{\prime}}{a_{1}}\left(a_{1} f+a_{0}\right)=\frac{f^{\prime} p_{1}(f)}{a_{1}} .
$$

Therefore, $g$ is a transcendental entire solution of

$$
w^{\prime} w+\frac{b_{0}-\alpha}{a_{1}}=\frac{s(z)}{a_{1}} e^{v(z)} .
$$

It follows from Theorem 1.1 that $\left(b_{0}-\alpha\right) / a_{1}=0$; therefore, $\alpha=b_{0}=q_{m}(0)$. This is a contradiction.

Next, if $n \geq 2$ and if $f^{\prime} p_{n}(f)+q_{m}(f)$ assumes $\alpha$ finitely many times, then there are a polynomial $u$ and a nonconstant polynomial $v$ such that

$$
f^{\prime}(z) p_{n}(f(z))+q_{m}(f(z))-\alpha=u(z) e^{v(z)} .
$$


Let $g(z)=f(z)+a_{n-1} / n a_{n}$. Therefore, $g$ is a transcendental entire function. Noting that the polynomial $p_{n}\left(w-a_{n-1} /\left(n a_{n}\right)\right)$ does not contain the term $w^{n-1}$,

$$
\begin{aligned}
f^{\prime} p_{n}(f)+q_{m}(f) & =g^{\prime} p_{n}\left(g-\frac{a_{n-1}}{n a_{n}}\right)+q_{m}\left(g-\frac{a_{n-1}}{n a_{n}}\right) \\
& =a_{n} g^{\prime} g^{n}+g^{\prime} A(g)+g B(g)+q_{m}\left(-\frac{a_{n-1}}{n a_{n}}\right),
\end{aligned}
$$

where $A, B$ are two polynomials, say,

$$
A(z)=a_{n-2}^{*} z^{n-2}+\cdots+a_{0}^{*} \quad \text { and } \quad B(z)=b_{m} z^{m-1}+b_{m-1}^{*} z^{m-2}+\cdots+b_{1}^{*} .
$$

Hence, $g$ is a transcendental entire solution of the equation

$$
w^{\prime} w^{n}+w^{\prime} \frac{A(w)}{a_{n}}+\frac{w B(w)}{a_{n}}+\frac{q_{m}\left(-a_{n-1} / n a_{n}\right)-\alpha}{a_{n}}=\frac{u}{a_{n}} e^{v} .
$$

It follows from Theorem 1.1 that there is a polynomial $s$ such that $g(z)=s(z) e^{v(z) /(n+1)}$ and, for all $z \in \mathbb{C}$,

$$
g^{\prime}(z) \frac{A(g(z))}{a_{n}}+\frac{g(z) B(g(z))}{a_{n}}+\frac{q_{m}\left(-a_{n-1} / n a_{n}\right)-\alpha}{a_{n}} \equiv 0 .
$$

It is clear that every nonconstant term in (3.14) is in the form of $u(z) e^{v(z)}$, where $u$ is a polynomial and $v$ is a nonconstant polynomial. Consequently, Lemma 2.2 gives $q_{m}\left(-a_{n-1} / n a_{n}\right)-\alpha=0$, which contradicts the assumption that $\alpha \neq q_{m}\left(-a_{n-1} / n a_{n}\right)$.

Now we consider the case that $f$ is of infinite order. It follows from Lemma 2.4 that there exists a sequence $\left\{z_{k}\right\}$ with $\lim _{k \rightarrow \infty} z_{k}=\infty$ such that $\left\{f_{k}(z)=f\left(z_{k}+z\right)\right\}_{k=1}^{\infty}$ is not normal at $z=0$. By Lemma 2.5, there exist a sequence of $\left\{w_{k}\right\}_{k=1}^{\infty},\left|w_{k}\right|<1$, and a positive sequence of $\rho_{k}, \rho_{k} \rightarrow 0$, such that

$$
h_{k}(z)=f_{k}\left(w_{k}+\rho_{k} z\right)=f\left(w_{k}+z_{k}+\rho_{k} z\right) \rightarrow h(z)
$$

spherically uniformly on any compact subset of $\mathbb{C}$, where $h(z)$ is a nonconstant entire function and its order satisfies $\rho(h) \leq 2$. Thus,

$$
\begin{aligned}
F_{k}(z):=\rho_{k}\left(f^{\prime}\left(w_{k}+z_{k}+\rho_{k} z\right)\left(a_{n} f\left(w_{k}+z_{k}+\rho_{k} z\right)^{n}+\cdots+a_{0}\right)\right) \\
\quad+\rho_{k}\left(b_{m} f\left(w_{k}+z_{k}+\rho_{k} z\right)^{m}+\cdots+b_{0}-\alpha\right) \\
=h_{k}^{\prime}(z)\left(a_{n} h_{k}(z)^{n}+\cdots+a_{0}\right)+\rho_{k}\left(b_{m} h_{k}^{m}(z)+\cdots+b_{0}-\alpha\right)
\end{aligned}
$$

converges to $h^{\prime}(z) p_{n}(h(z))$ spherically uniformly on any compact subset of $\mathbb{C}$.

We claim that $h^{\prime}(z) p_{n}(h(z))$ does not assume zero if $f^{\prime} p_{n}(f)+q_{m}(f)$ assumes $\alpha$ finitely many times. In fact, if $h^{\prime}(z) p_{n}(h(z))$ has a zero, say $\zeta_{0}$, then, by Hurwitz's theorem, there are a sequence of complex numbers $\left\{\zeta_{k}\right\}$ and a sufficiently large integer $N$ such that $F_{k}\left(\zeta_{k}\right)=0(k \geq N)$ and $\zeta_{k} \rightarrow \zeta_{0}$. Hence,

$$
\begin{array}{r}
f^{\prime}\left(w_{k}+z_{k}+\rho_{k} z\right)\left(a_{n} f\left(w_{k}+z_{k}+\rho_{k} z\right)^{n}+\cdots+a_{0}\right) \\
+b_{m} f\left(w_{k}+z_{k}+\rho_{k} z\right)^{m}+\cdots+b_{0}-\alpha=0
\end{array}
$$


where $\xi_{k}=w_{k}+z_{k}+\rho_{k} \zeta_{k}$. Since $\left|w_{k}\right|<1$,

$$
\lim _{k \rightarrow \infty} z_{k}=\infty, \quad \lim _{k \rightarrow \infty} \rho_{k}=0 \quad \text { and } \quad \lim _{k \rightarrow \infty} \zeta_{k}=\zeta_{0}
$$

we can choose subsequences $\left\{z_{k_{j}}\right\},\left\{w_{k_{j}}\right\}$ and $\left\{\rho_{k_{j}}\right\}$ such that

$$
\left|z_{k_{j}}-z_{k_{l}}\right|>3 \quad \text { when } k_{j} \neq k_{l}, \quad\left|w_{k_{j}}\right|<1 \quad \text { and } \quad\left|\rho_{k_{j}} \zeta_{k_{j}}\right|<\frac{1}{2} \text {. }
$$

Thus, $\xi_{k_{j}}=w_{k_{j}}+z_{k_{j}}+\rho_{k_{j}} \zeta_{k_{j}}(j=1,2, \ldots)$ are distinct zeros of $f^{\prime} p_{n}(f)+q_{m}(f)-a$. This is a contradiction.

Hence, we have proved that if $f^{\prime} p_{n}(f)+q_{m}(f)$ assumes a complex value $a$ finitely many times, then $h^{\prime}(z) p_{n}(h(z)) \neq 0$ for all $z \in \mathbb{C}$. Consequently, $h$ is a transcendental entire function of finite order and there is a nonconstant polynomial $v$ such that

$$
h^{\prime} p_{n}(h)=h^{\prime}\left(a_{n} h^{n}+a_{n-1} h^{n-1}+\cdots+a_{0}\right)=e^{v(z)} .
$$

Let $H(z)=h(z)+a_{n-1} / n a_{n}$. Then

$$
Q_{n}(h)=\sum_{j=0}^{n} a_{j}\left(H-\frac{a_{n-1}}{n a_{n}}\right)^{j}=a_{n} H^{n}+A(H),
$$

where $A(H)$ is a polynomial of $H$ with degree not greater than $n-2$. Hence, (3.15) turns out to be

$$
H^{\prime} H^{n}+\frac{1}{a_{n}} A(H) H^{\prime}=\frac{1}{a_{n}} e^{v(z)} .
$$

It follows from Theorem 1.1 that $A(H) H^{\prime} \equiv 0$; therefore, $A(H) \equiv 0$. Hence, (3.16) gives

$$
p_{n}(z)=a_{n}\left(z+\frac{a_{n-1}}{n a_{n}}\right)^{n} .
$$

Let $g(z)=1 / x\left(f(z)+a_{n-1} / n a_{n}\right)$, where $x$ is a complex number such that $x^{n+1} a_{n}=1$. Thus,

$$
\begin{aligned}
f^{\prime} p_{n}(f)+q_{m}(f) & =x g^{\prime} p_{n}\left(x g-\frac{a_{n-1}}{n a_{n}}\right)+q_{m}\left(x g-\frac{a_{n-1}}{n a_{n}}\right) \\
& =a_{n} x^{n+1} g^{\prime} g^{n}+q_{m}\left(x g-\frac{a_{n-1}}{n a_{n}}\right)=g^{\prime} g^{n}+q_{m}\left(x g-\frac{a_{n-1}}{n a_{n}}\right)
\end{aligned}
$$

assumes $\alpha$ finitely many times. On the other hand, Theorem 1.3 shows that

$$
g^{\prime} g^{n}+q_{m}\left(x g-\frac{a_{n-1}}{n a_{n}}\right)
$$

which is the right-hand side of (3.17), assumes every $\alpha \neq q_{m}\left(-a_{n-1} /\left(n a_{n}\right)\right)$ infinitely many times. This is impossible. Therefore, $f^{\prime} p_{n}(f)+q_{m}(f)$ takes every $\alpha \neq$ $q_{m}\left(-a_{n-1} /\left(n a_{n}\right)\right)$ infinitely many times.

Furthermore, if $f^{\prime} p_{n}(f)+q_{m}(f)$ takes $q_{m}\left(-a_{n-1} /\left(n a_{n}\right)\right)$ finitely many times and $f$ is of infinite order, then, by (3.17), $g^{\prime} g^{n}+q_{m}\left(x g-a_{n-1} / n a_{n}\right)$ takes $q_{m}\left(-a_{n-1} /\left(n a_{n}\right)\right)$ 
finitely many times. It follows from Theorem 1.3 that the polynomial $q_{m}(x g-$ $\left.a_{n-1} / n a_{n}\right)$ is the constant $q_{m}\left(-a_{n-1} / n a_{n}\right)$. Therefore, (3.17) becomes

$$
p_{n}(f)=\frac{g^{n}}{x}=a_{n}\left(f(z)+\frac{a_{n-1}}{n a_{n}}\right)^{n},
$$

since $f^{\prime}=x g^{\prime}$ and $q_{m}(f)=q_{m}\left(-a_{n-1} /\left(n a_{n}\right)\right)$. This means that $p_{n}(z)=a_{n}\left(z+a_{n-1} / n a_{n}\right)^{n}$, that is, a complete power function.

If $f^{\prime} p_{n}(f)+q_{m}(f)$ takes $q_{m}\left(-a_{n-1} /\left(n a_{n}\right)\right)$ finitely many times and $f$ is of finite order, then we consider two cases: $q_{m}(z)$ is a constant polynomial and $q_{m}(z)$ is a nonconstant polynomial.

When $q_{m}(z)$ is a constant, then $\alpha=q_{m}\left(-a_{n-1} / n a_{n}\right)=b_{0}$ and $B(z) \equiv 0$, where $B(z)$ is defined in (3.14). Therefore, (3.14) gives $A(z) \equiv 0$. Thus, (3.13) becomes

$$
p_{n}(f)=a_{n} g^{n}=a_{n}\left(f+\frac{a_{n-1}}{n a_{n}}\right)^{n},
$$

since $f^{\prime}=g^{\prime}$ and $q_{m}(f)=q_{m}\left(-a_{n-1} /\left(n a_{n}\right)\right)$. Therefore, $p_{n}$ is a complete power function.

When $q_{m}(z)$ is a nonconstant polynomial with degree $m \geq 1$ or $b_{m} \neq 0$, then, substituting $g(z)=s(z) e^{v(z) /(n+1)}$ into (3.14) and by Lemma 2.2, $\alpha=q_{m}\left(-a_{n-1} / n a_{n}\right)$ and

$$
a_{m-1}^{*}\left(s^{\prime}(z)+s(z) \frac{v^{\prime}(z)}{n+1}\right)+b_{m} s(z) \equiv 0,
$$

which is the coefficient of the term $e^{m v(z) /(n+1)}$, since $n \geq m+1$. If $a_{m-1}^{*}=0$, then $b_{m}=0$, which is a contradiction. Hence, $a_{m-1}^{*} \neq 0$ and

$$
\left(\frac{a_{m-1}^{*} v^{\prime}(z)}{n+1}+b_{m}\right) s(z)+a_{m-1}^{*} s^{\prime}(z) \equiv 0 .
$$

Thus, $a_{m-1}^{*} v^{\prime}(z) /(n+1)+b_{m} \equiv 0$ and $s^{\prime}(z) \equiv 0$. It follows that $s(z)$ is a constant, say $C^{\prime}$, and $v(z)$ is a linear polynomial, say $D z+D^{\prime}$, that is, $g(z)=C e^{D z}$ for some constants $C, D$. Hence, $f(z)=C e^{D z}+a_{n-1} / n a_{n}$. Thus, the proof of the theorem is complete.

By Theorem 1.5, we have the following results.

COROLlary 3.2. Let $f$ be a transcendental entire function and $p_{n}(z)$ a polynomial with degree, $n \geq 1$, as in the theorem. Then $f^{\prime} p_{n}(f)$ assumes every nonzero complex number $\alpha$ infinitely many times. On the other hand, if $f^{\prime} p_{n}(f)$ takes 0 finitely many times, then $p_{n}(z)=a_{n}\left(z+a_{n-1} / n a_{n}\right)^{n}, f+a_{n-1} / n a_{n}$ and $f^{\prime}$ have only finitely many zeros.

Corollary 3.3. Let $\mathfrak{i}$ be a family of holomorphic functions in a domain D. Suppose that $f^{\prime} Q_{n}(f)$ does not assume a complex number $a \neq 0$ for every function $f \in \mathfrak{F}$, where $Q_{n}(f)$ is a polynomial with degree $n \geq 1$. Then $\mathfrak{F}$ is a normal family in the domain $D$.

\section{Acknowledgement}

The authors would like to thank the referee for his/her comments and suggestions. 


\title{
References
}

[1] A. Alotaibi and J. K. Langley, 'The separation of zeros of solutions of higher order linear differential equations with entire coefficients', Results Math. 63(3-4) (2013), 1365-1373.

[2] H. Chen, 'Yosida functions and Picard values of integral functions and their derivatives', Bull. Aust. Math. Soc. 54(3) (1996), 373-381.

[3] W. Cherry and Z. Ye, Nevanlinna's Theory of Value Distribution, Springer Monographs in Mathematics (Springer, Berlin, 2001).

[4] M. Chuaqui, J. Gröhn, J. Heittokangas and J. Rättyä, 'Zero separation results for solutions of second order linear differential equations', Adv. Math. 245 (2013), 382-422.

[5] J. Clunie, 'On a result of Hayman', J. Lond. Math. Soc. 42 (1967), 389-392.

[6] F. Gackstatter and I. Laine, 'Zur Theorie der gewöhnlichen Differentialgleichungen im Komplexen', Ann. Polon. Math. 38(3) (1980), 259-287.

[7] W. K. Hayman, 'Picard values of meromorphic functions and their derivatives', Ann. of Math. (2) 70(2) (1959), 9-42.

[8] W. K. Hayman, Meromorphic Functions (Clarendon Press, Oxford, 1964).

[9] Y. He and I. Laine, 'The Hayman-Miles theorem and the differential equation $\left(y^{\prime}\right)^{n}=R(z, y)^{\text {', }}$ Analysis 10(4) (1990), 387-396.

[10] J. Heittokangas and J. Rättyä, 'Zero distribution of solutions of complex linear differential equations determines growth of coefficients', Math. Nachr. 284(4) (2011), 412-420.

[11] I. Laine, 'Complex differential equations', in: Handbook of Differential Equations: Ordinary Differential Equations, Vol. IV (Elsevier/North-Holland, Amsterdam, 2008), 269-363.

[12] P. Li, 'Entire solutions of certain type of differential equations II', J. Math. Appl. 375 (2011), 310-319.

[13] L. W. Liao, C. C. Yang and J. J. Zhang, 'On meromorphic solutions of certain type of nonlinear differential equations', Ann. Acad. Sci. Fenn. Math. 38 (2013), 581-593.

[14] L. W. Liao and Z. Ye, 'A class of second order differential equations', Israel J. Math. 146 (2005), 281-301.

[15] E. Mues, 'Zur Wertverteilung von Differentialpolynomen', Arch. Math. (Basel) 32(1) (1979), $55-67$.

[16] Y. Xu, F. Wu and L. W. Liao, 'Picard values and normal families of meromorphic functions', Proc. Roy. Soc. Edinburgh Sect. A 139(5) (2009), 1091-1099.

[17] C. C. Yang and Z. Ye, 'Estimates of the proximate function of differential polynomials', Proc. Japan Acad. Ser. A Math. Sci. 83(4) (2007), 50-55.

[18] C. C. Yang and H. X. Yi, Uniqueness Theory of Meromorphic Functions (Science Press and Kluwer Academic, Beijing, 2003).

[19] L. Zalcman, 'Normal families: new perspectives', Bull. Amer. Math. Soc. 35 (1998), 215-230.

[20] Z. Zhang and W. Li, 'The derivatives of polynomials of entire and meromorphic functions', Chinese Ann. Math. Ser. A 15(2) (1994), 217-223 (in Chinese).

[21] J. Zhang and L. Liao, 'Admissible meromorphic solutions of algebraic differential equations', J. Math. Anal. Appl. 397(1) (2013), 225-232.

LIANG-WEN LIAO, Department of Mathematics, Nanjing University, Nanjing, PR China

e-mail:maliao@nju.edu.cn

\author{
ZHUAN YE, Department of Mathematical Sciences, \\ Northern Illinois University, DeKalb, IL 60115, USA \\ e-mail: ye@math.niu.edu \\ and
}

Department of Mathematics, Taiyuan University of Technology, Taiyuan, Shanxi, PR China 